\title{
Carcinóide Pulmonar: Análise de Experiência Institucional e Fatores Prognóstico
}

\author{
Pulmonary Carcinoid: Analysis of a Single Institutional Experience and \\ Prognostic Factors
}

Sílvia da Silva CORREIA ${ }^{1}$, Carlos PINTO², João BERNARDO²

Acta Med Port 2014 Nov-Dec;27(6):749-754

RESUMO

Introdução: Os tumores carcinóides pulmonares são tumores raros com origem nas células neuro-endócrinas do pulmão. Classificam-se de acordo com os critérios da OMS em carcinóides típicos ou atípicos. Quando comparados com outros tipos de neoplasia pulmonar, os tumores carcinóides apresentam melhor prognóstico.

Objetivos: Caracterização dos doentes com diagnóstico histológico de tumor carcinóide observados numa instituição. Análise dos fatores que influenciaram o prognóstico.

Material e Métodos: Análise retrospetiva incluindo todos os doentes com diagnóstico histológico de tumor carcinóide pulmonar durante um período de 11 anos numa instituição. Os tumores foram classificados em típicos e atípicos de acordo com a classificação da Organização Mundial de Saúde de 2004. O estadiamento foi feito com base na classificação TNM de 2009 para o carcinoma do pulmão de não pequenas células: T (Tumor); N (Ganglionar); M (Metástase).

Resultados: Foram incluídos 59 doentes: 53 carcinóides típicos e seis carcinóides atípicos. Destes, $90 \%$ foram submetidos a cirurgia. O follow-up médio foi de 57 meses. A mortalidade operatória foi de $2 \%(n=1)$ tratando-se de cirurgia paliativa para um doente em estádio IV. Em 49 doentes não se verificou envolvimento ganglionar (N0), um doente apresentava doença N1, oito doença N2 e um doente doença N3. A sobrevivência global aos cinco anos foi de $79,2 \%$ : $80,2 \%$ nos carcinóides típicos e $66,7 \%$ nos carcinóides atípicos $(p<0,05)$. Nos doentes $T 1$, a sobrevivência foi de $88,1 \%$ e de $58,2 \%$ nos T2-T4 $(p<0,01)$. Nos doentes N0 a sobrevivência aos cinco anos de $89,7 \%$ e de $36 \%$ para os doentes N1-N3 $(p<0,001)$. Os doentes com doença M0 apresentaram uma sobrevivência aos cinco anos de $85,9 \%$ sendo de $0 \%$ nos doentes M1 $(p<0,01)$. Dos 11 doentes que necessitaram de quimioterapia adjuvante, $45,4 \%$ eram carcinóides atípicos.

Discussão: Na nossa série, a cirurgia no tumor carcinóide pulmonar demonstrou-se segura, com uma baixa taxa de complicações no pós-operatório. Observou-se pior prognóstico em doentes com tumor carcinóide atípico, tumores com mais de $3 \mathrm{~cm}$, com envolvimento ganglionar ou presença de metástase. A sobrevivência aos cinco anos nos tumores carcinóides típicos foi excelente (80,2\%), correspondente à encontrada na literatura. No tumor carcinóide atípico, a sobrevivência aos cinco anos foi de $66,7 \%$ também concordante com os dados obtidos em estudos anteriores.

Conclusões: Os tumores carcinóides pulmonares são na maioria carcinóides típicos com uma excelente sobrevivência a longo prazo. A cirurgia de ressecção é o tratamento de eleição nestes doentes. Os fatores relacionados com um pior prognóstico foram o subtipo histológico (carcinóides típicos versus carcinóides atípicos), o tamanho do tumor, o envolvimento ganglionar e a presença de metástases.

Palavras-chave: Neoplasias do Pulmão; Tumor Carcinóide; Estadiamento.

\section{ABSTRACT}

Introduction: Carcinoid tumors of the lung are rare neoplasms of neuroendocrine origin. According to the World Health Organization, they can be classified into typical carcinoids and atypical carcinoids. The outcome, when compared to other lung neoplasms is usually favorable.

Objectives: To characterize the population of patients with a diagnosis of carcinoid tumor, treated in a single institution and analyze the prognostic factors.

Material and Methods: Retrospective analysis including all the patients with histological diagnosis of lung carcinoid tumor during an 11 year period, in a single institution. The tumors were classified according to the World Health Organization classification of carcinoids tumors in 2004. Staging was made according to the TNM classification of 2009 for non-small lung cancer: T (Tumor); N (Node); M (metastasis).

Results: 59 patients were evaluated, including 53 with typical carcinoids and 6 with atypical carcinoid. $90 \%$ of the patients were submitted to surgery. The mean follow-up period was 57 months and the early post-operative mortality rate was $2 \%$ (one single case of palliative surgery). Histologic staging showed 49 patients in stage N0, one N1, eight N2 and one N3. The 5-year survival was $79.2 \%$ : $80.2 \%$ for typical carcinoids and $66.7 \%$ for atypical carcinoid $(p<0.05)$. The 5 -year survival was $88.1 \%$ in T1 patients and $58.2 \%$ in T2-T4 patients $(p<0.01)$. In N0 patients, the 5 -year survival was $89.7 \%$ while in N1-N3 it was $36 \%$ $(p<0.001)$. The 5 -year survival was $85.9 \%$ in M0 disease and $0 \%$ in M1 disease $(p<0.01)$. Of the 11 patients who were submitted to adjuvant chemotherapy, $45.4 \%$ had atypical tumors.

Discussion: In our sample, surgical treatment was safe, with a low postoperative complication rate. The prognosis was worse for atypical tumors, tumors with more than $3 \mathrm{~cm}$, tumors with nodal involvement or metastasis. The five-year survival for typical carcinoid was excellent $(80.2 \%)$, in agreement with the literature. For atypical carcinoid, the five-year was $66.7 \%$, also similar to previous studies. Conclusions: In our institution, most of the lung carcinoids are typical and have an excellent long term survival. The mainstay of treatment is surgical resection. The factors that were related to a poor prognosis were the histological subtype (typical carcinoids versus

1. Serviço de Pneumologia. Unidade Local de Saúde. Guarda. Portugal.

2. Centro de Cirurgia Cardiotorácica. Centro Hospitalar e Universitário de Coimbra. Coimbra. Portugal.

Recebido: 31 de Março de 2014 - Aceite: 21 de Julho de 2014 | Copyright @ Ordem dos Médicos 2014 
atypical carcinoids), the size of the tumor, the mediastinal lymphatic involvement and the presence of metastasis.

Keywords: Lung Neoplasms; Carcinoid Tumor; Neoplasm Staging.

\section{INTRODUÇÃO}

Os tumores carcinóides pulmonares são tumores bem diferenciados com origem nas células neuro-endócrinas do pulmão. São tumores raros, representando 0,4 a $3 \%$ dos tumores pulmonares ressecados e 23 a $30 \%$ de todos os tumores neuroendócrinos. ${ }^{1,2}$ São classificados em típicos ou atípicos de acordo com os critérios histológicos da Organização Mundial de Saúde de 2004: o tumor carcinóide típico é um tumor com $<2$ mitoses $/ 2 \mathrm{~mm}^{2}$, sem necrose e um tamanho superior a $5 \mathrm{~mm}$. Os carcinóides atípicos distinguem-se por um número de mitoses de dois a 10 , existência de necrose ou distorção da arquitetura. ${ }^{3} \mathrm{Em}$ termos clínicos, são geralmente tumores centrais que cursam com obstrução brônquica. Os sintomas mais frequentes são a tosse ou hemoptise mas muitos doentes são assintomáti$\cos ^{4,5}$ Podem ocorrer em qualquer idade e não existe diferença entre os dois sexos. Relativamente ao prognóstico, quando comparados com outros carcinomas pulmonares de não pequenas células, os carcinóides apresentam geralmente um prognóstico favorável após ressecção cirúrgica. Os tumores carcinóides típicos raramente metastizam e apresentam uma sobrevivência aos cinco anos que varia entre os 87 a $100 \%{ }^{6.7}$ Pelo contrário, os tumores carcinóides atípicos apresentam um risco maior de metastização e a sobrevivência aos cinco anos varia entre os 25 a $69 \%^{8,9}$ Poucos estudos avaliaram o prognóstico dos tumores carcinóides de acordo com o subtipo histológico, tipo de cirurgia, tamanho do tumor, envolvimento ganglionar e presença de metástases, segunda a nova classificação TNM.10,11 O objetivo do presente estudo é assim a caracterização dos doentes com diagnóstico de tumor carcinóide e a análise dos fatores que influenciaram o prognóstico num centro terciário.

\section{MATERIAL E MÉTODOS}

Foi realizado um estudo retrospetivo incluindo todos os doentes com um diagnóstico de tumor carcinóide pulmonar realizado no Centro Hospitalar e Universitário de Coimbra no período de 1999 a 2010. Os dados foram obtidos através da consulta do processo clínico de cada doente e incluíam variáveis epidemiológicas, clínicas (anamnese com ênfase nos sintomas e exame físico), intervenções (incluindo broncoscopia, biópsia transtorácica e cirurgias) e dados relativos ao prognóstico. O diagnóstico histológico foi confirmado por um anatomo-patologista com experiência em carcinomas neuroendócrinos e todos foram classificados de acordo com os critérios da Organização Mundial Saúde. ${ }^{3} \mathrm{O}$ estadiamento foi feito com base na classificação TNM de 2009 para o carcinoma do pulmão de não pequenas células: T (Tumor); N (Ganglionar); M (Metástase). ${ }^{12,13}$ A análise estatística foi realizada recorrendo ao software IBM SPSS, versão 17. A sobrevivência aos cinco anos foi estimada pelo método de Kaplan e Meier. A significância estatística foi considerada para valores de $p<0,05$.

\section{RESULTADOS}

Foram incluídos 59 doentes: 33 homens e 26 mulheres com uma idade média de $54,5 \pm 14,5$ anos (16 - 79) (Fig. 1).

De acordo com a classificação histológica, 53 doentes apresentavam um tumor carcinóide típico (CT) e seis, um carcinóide atípico (CA). A descrição da amostra pode ser consultada na Tabela 1.

A maioria dos doentes apresentava como sintoma principal a tosse $(47 \%), 34 \%$ toracalgia, $29 \%$ dispneia, $15 \%$ hemoptise e $2 \%$ síndrome de Cushing. Em 29\% dos casos não se observaram sintomas. Radiologicamente a neoplasia foi identificada na tomografia computorizada do tórax sob a forma de um nódulo central em $51 \%$ dos doentes, de um nódulo periférico em $34 \%$ e sob a forma de atelectasia em $15 \%$. Dentro dos nódulos centrais $(n=30)$, a maioria estava localizada no lobo superior e inferior direito. A localização tumoral pode ser observada na Tabela 2.

Quando comparados os sintomas com a localização do tumor (central vs. periférica), a hemoptise foi mais frequente nos tumores centrais $(p=0,012)$. Não obtivemos diferenças estatisticamente significativas no que diz respeito à dispneia, tosse e toracalgia.

O estadiamento tumoral encontra-se resumido na Tabela 3. Em 49 doentes, não se verificou envolvimento

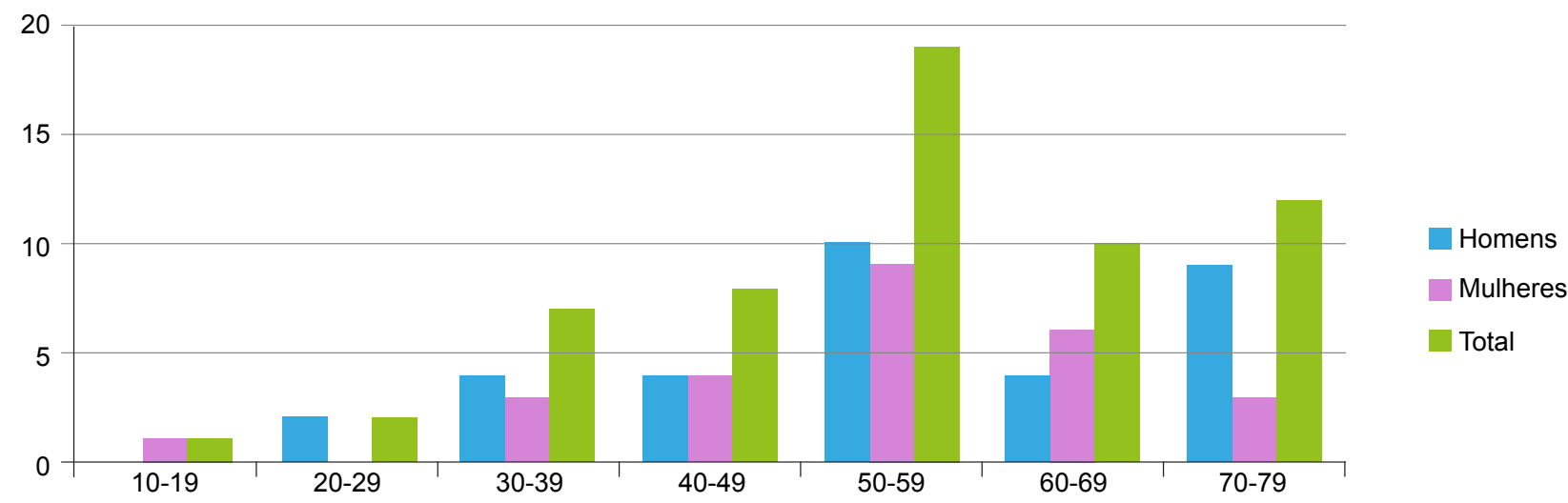

Figura 1 - Distribuição dos doentes por intervalo de idade e género 
Tabela 1 - Características histológicas, clínicas e radiológicas

\begin{tabular}{|c|c|c|}
\hline \multicolumn{3}{|c|}{ Características histológicas, clínicas e radiológicas } \\
\hline Histológica & $n$ & $\%$ \\
\hline Carcinoíde típico & 53 & $89,8 \%$ \\
\hline Carcinoíde atípico & 6 & $10,2 \%$ \\
\hline \multicolumn{3}{|l|}{ Sintomas } \\
\hline Tosse & 28 & $47,0 \%$ \\
\hline Toracalgia & 20 & $34,0 \%$ \\
\hline Dispneia & 17 & $29,0 \%$ \\
\hline Hemoptise & 9 & $15,0 \%$ \\
\hline Síndrome de Cushing & 1 & $2,0 \%$ \\
\hline Assintomático & 17 & $29,0 \%$ \\
\hline \multicolumn{3}{|c|}{ Apresentação radiológica } \\
\hline Nódulo central & 30 & $51,0 \%$ \\
\hline Nódulo periférico & 20 & $34,0 \%$ \\
\hline Atelectasia & 9 & $15,0 \%$ \\
\hline
\end{tabular}

Tabela 2 - Localização dos tumores carcinóides brônquicos

\begin{tabular}{lcc}
\hline Localização dos tumores carcinóide brônquicos & $n$ & $\%$ \\
Local & 2 & $3,4 \%$ \\
\hline Brônquio principal direito & 5 & $8,5 \%$ \\
Brônquio principal esquerdo & 11 & $18,7 \%$ \\
Lobo superior direito & 10 & $16,9 \%$ \\
Lobo médio & 10 & $16,9 \%$ \\
Lobo inferior direito & 13 & $22 \%$ \\
Lobo superior esquerdo & 8 & $13,6 \%$ \\
Lobo inferior esquerdo & $\mathbf{1 0 0 \%}$ \\
TOTAL & $\mathbf{5 9}$ \\
\hline
\end{tabular}

ganglionar (N0). No grupo dos CT ( $n=53$ doentes), 47 doentes (89\%) não apresentavam envolvimento ganglionar (N0), enquanto que no grupo dos CA (6 doentes), a maioria apresentava doença N2 ou N3 (50\%).

Relativamente ao diagnóstico, a broncoscopia flexível foi realizada em $69 \%(n=41)$ dos doentes sendo que em $90 \%$ destes foi visualizado o tumor (classificado assim como central). Nos tumores considerados centrais $(n=37)$, $83 \%$ eram CT. Todos foram submetidos a biópsia brônquica: em $76 \%$ foi identificado um tumor carcinoíde, em $21 \%$ o resultado histopatológico foi inconclusivo e em $3 \%$ revelou outro subtipo de neoplasia pulmonar. A biópsia transtorácica por agulha foi efectuada em $12 \%$ dos doentes sendo que em $86 \%$ foi identificado um tumor carcinóide e em $14 \%$ revelou outro tipo de neoplasia pulmonar.

A maioria dos doentes ( $n=53 ; 90 \%)$ foi sujeita a cirurgia. A causa mais frequente para o tratamento não cirúrgico foi a presença de doença metastizada (quatro doentes); um doente foi submetido a terapêutica endoscópica e um doente recusou tratamento cirúrgico. O procedimento cirúrgico consistiu em lobectomia em $64 \%$ dos doentes, ressecção sublobar atípica em $26 \%$, pneumectomia em $7 \%$ e bilobectomia em $4 \%$. O procedimento incluiu linfadedectomia mediastínica radical em todos os doentes. A mortalidade operatória foi de $2 \%$ (um doente) tratando-se de cirurgia paliativa para um doente de 57 anos de idade e 
Tabela 3 - Estadiamento dos doentes com tumor carcinoíde pulmonar

\begin{tabular}{|c|c|c|c|}
\hline Variável & & $n$ & $\%$ \\
\hline \multirow{5}{*}{ Tumor primário } & T1a & 25 & $42,4 \%$ \\
\hline & $\mathrm{T} 1 \mathrm{~b}$ & 16 & $27,1 \%$ \\
\hline & $\mathrm{T} 2 \mathrm{a}$ & 10 & $17 \%$ \\
\hline & $\mathrm{T} 2 \mathrm{~b}$ & 2 & $3,4 \%$ \\
\hline & T3-T4 & 6 & $10,1 \%$ \\
\hline \multirow{4}{*}{ Envolvimento ganglionar } & No & 49 & $83 \%$ \\
\hline & N1 & 1 & $1,7 \%$ \\
\hline & $\mathrm{N} 2$ & 8 & $13,6 \%$ \\
\hline & N3 & 1 & $1,7 \%$ \\
\hline \multirow{2}{*}{ Metastização } & M0 & 55 & $93 \%$ \\
\hline & M1 & 4 & $7 \%$ \\
\hline \multirow{4}{*}{ Estádio } & I & 49 & $83 \%$ \\
\hline & II & 1 & $1,7 \%$ \\
\hline & III & 5 & $8,5 \%$ \\
\hline & IV & 4 & $6,8 \%$ \\
\hline
\end{tabular}

Tabela 4 - Sobrevivência dos doentes com tumor carcinoíde pulmonar

\begin{tabular}{|c|c|c|c|c|c|}
\hline & & $n$ & Mortalidade & $\begin{array}{l}\text { Sobrevivência } \\
\text { aos } 5 \text { anos }\end{array}$ & $p$ \\
\hline \multirow{2}{*}{ Sexo } & Masculino & 33 & $33,3 \%$ & $71,2 \%$ & \\
\hline & Feminino & 26 & $15,4 \%$ & $90,4 \%$ & $>0,05$ \\
\hline \multirow{3}{*}{ Idade } & $16-40$ & 11 & $9 \%$ & $90,9 \%$ & \\
\hline & $41-60$ & 30 & $23,3 \%$ & $77,1 \%$ & \\
\hline & $>60$ & 18 & $38,9 \%$ & $76,2 \%$ & $>0,05$ \\
\hline \multirow{2}{*}{ Subtipo } & Típico & 53 & $20,8 \%$ & $80,2 \%$ & \\
\hline & Atípico & 6 & $66,7 \%$ & $66,7 \%$ & $<0,05$ \\
\hline \multirow{2}{*}{ Tumor } & $\mathrm{T} 1$ & 41 & $14,6 \%$ & $88,1 \%$ & \\
\hline & $\mathrm{T} 2-\mathrm{T} 4$ & 18 & $50 \%$ & $58,2 \%$ & $<0,01$ \\
\hline \multirow{2}{*}{ Envolvimento ganglionar } & No & 49 & $16,3 \%$ & $89,7 \%$ & \\
\hline & N1-N3 & 10 & $70 \%$ & $36,0 \%$ & $<0,001$ \\
\hline
\end{tabular}

metástase pulmonar contra lateral única. A cirurgia consistiu em exérese de lesão hilar direita de grandes dimensões que comprimia o mediastino e a aurícula direita. Observaram-se complicações no pós-operatório em $24 \%$ dos doentes: derrame pleural $(n=6 ; 10 \%)$, pneumotórax $(n=5 ; 8 \%)$, empiema $(n=1 ; 2 \%)$ e hemorragia $(n=1 ; 2 \%)$. O follow-up médio foi de 57 meses (0 - 144). Registaram-se 15 mortes, nove relacionadas com a neoplasia e seis por causas não relacionadas (três doentes faleceram com enfarte agudo do miocárdio e três com acidente vascular cerebral isquémico). Nos falecidos, 11 doentes apresentavam CT e quatro CA. Oito doentes apresentaram metastização: dois CA e seis CT. Nos CT, quatro tiveram metastização pulmonar e dois metastização hepática. Nos CA a metastização foi 


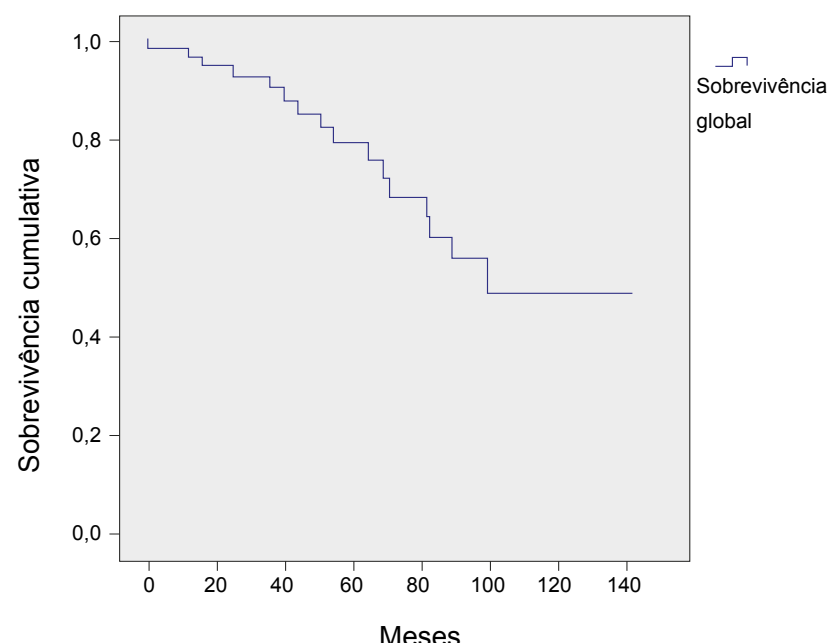

Figura 2 - Curva de sobrevivência global nos tumores carcinóides

exclusivamente pulmonar.

Os doentes submetidos a ressecção sublobar atípica não apresentaram diferenças na sobrevivência em relação aos doentes submetidos a ressecção lobar (Log-rank; $p=$ $0,359)$.

A sobrevivência global aos cinco anos foi de $79,2 \%$ (Tabela 4): $71,2 \%$ no sexo masculino e $90,4 \%$ no sexo feminino $(p>0,05)$. De acordo com o escalão etário (16-40 anos; 41-60 anos; $>60$ anos), a sobrevivência aos cinco anos de $90,9 \%, 77,1 \%$ e $76,2 \%$ respectivamente $(p>0,05)$. A sobrevivência aos cinco anos nos CT foi de $80,2 \%$ e $66,7 \%$ nos CA $(p<0,05)$. Nos doentes com tumor inferior a $3 \mathrm{~cm}$ (T1), a sobrevivência aos cinco anos foi de $88,1 \%$ sendo de $58,2 \%$ nos restantes doentes (T2-T4) $(p<0,01)$. De acordo com o envolvimento ganglionar, os doentes com doença N0 apresentaram uma sobrevivência aos cinco anos de $89,7 \%$, sendo de $36 \%$ nos restantes doentes (N2-N3) ( $p$ $<0,001)$. Os doentes com doença M0 apresentaram uma sobrevivência aos cinco anos de $85,9 \%$ sendo de $0 \%$ nos doentes M1 $(p<0,01)$.

Estes resultados encontram-se apresentados na Tabela 3. A análise da sobrevivência através de curvas de Kaplan-Meier é documentada nas Fig.s 2 e 3.

Dos 11 doentes que necessitaram de quimioterapia adjuvante, $45,4 \%$ eram CA. A quimioterapia consistiu em cisplatino e etoposido em todos os casos.

\section{DISCUSSÃO}

Os carcinóides broncopulmonares são tumores que surgem em idades mais jovens que os outros tipos de neoplasia pulmonar. Na nossa amostra estes ocorreram predominantemente na quinta década, o que corresponde ao descrito na literatura. ${ }^{3,12} \mathrm{O}$ rácio homens/mulheres foi de 1,26 semelhante ao encontrado por Filosso et al (rácio de 1,33 ) e Cardillo et al (rácio de 1,11). ${ }^{14-16}$ A maioria dos doentes era sintomática $(71 \%)$ o que corresponde a uma percentagem ligeiramente superior à previamente descrita. ${ }^{16,17}$ Em termos de localização, a maioria dos tumores estavam localizados nos lobos superiores, ao contrário de algumas

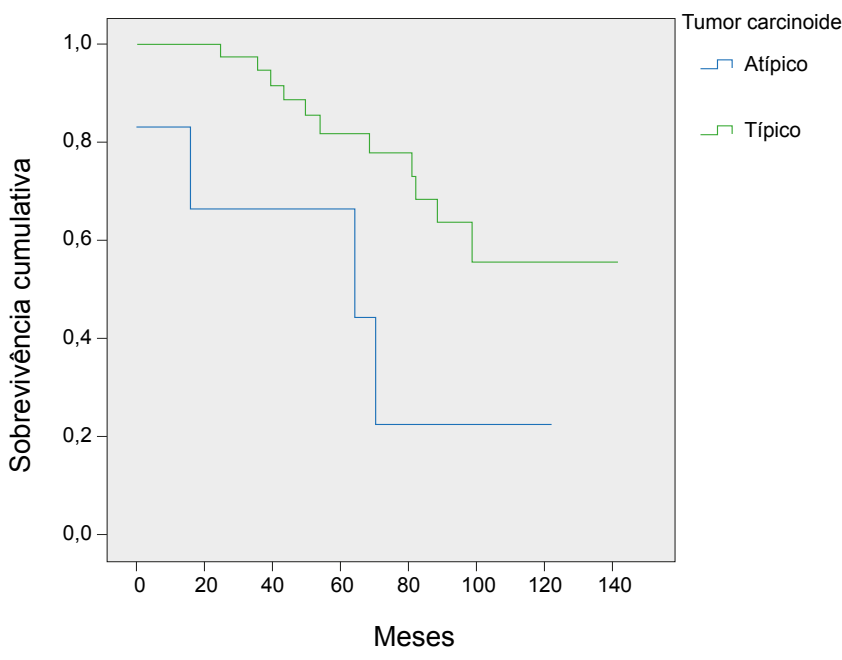

Figura 3 - Curva de sobrevivência nos doentes com CT e CA

séries que apontam o lobo médio como o local favorito para o aparecimento dos tumores carcinóides pulmonares. ${ }^{18}$

A broncofibroscopia é um exame essencial na avaliação dos doentes com tumor carcinoíde por este se apresentar frequentemente sob a forma de um tumor central. Na nossa série, $90 \%$ eram tumores centrais o que permite a realização de biópsias brônquicas e a obtenção de um diagnóstico atempado. No entanto, o diagnóstico histológico obtido com a biópsia brônquica exige confirmação com o da peça operatória. Como foi descrito, em $21 \%$ o resultado histopatológico da biópsia brônquica revelou alterações inespecíficas e em $3 \%$ revelou outro subtipo de neoplasia pulmonar. Já a biópsia transtorácica por agulha revela-se importante nos doentes com tumor periférico, não acessível à broncofibroscopia.

A maioria dos tumores considerados centrais (tumor endoscopicamente visível) eram tumores CT. Embora não se consiga explicar a razão dessa associação, esta já foi demonstrada noutras séries anteriores. ${ }^{1}$

O tratamento de eleição do tumor carcinóide pulmonar é a ressecção cirúrgica. Dos 53 doentes submetidos a cirurgia, 6 apresentavam metástases ganglionares (11\%). A ressecção sublobar atípica realizada nos doentes com CT central e sem metástases ganglionares permite uma óptima sobrevivência a longo prazo. ${ }^{2,17}$ Uma linfadenectomia mediastínica radical deve ser sempre realizada embora existam poucos estudos na literatura sobre este tema. ${ }^{19,20}$

Na nossa série, a cirurgia no tumor carcinóide pulmonar demonstrou-se segura, com uma baixa taxa de complicações no pós-operatório.

Os carcinóides atípicos apresentam um maior risco de metastização e pior prognóstico que os CT. Observou-se pior prognóstico em doentes com CA, tumores com mais de $3 \mathrm{~cm}$, com envolvimento ganglionar e com presença de metástase. A sobrevivência aos cinco anos nos CT foi excelente $(80,2 \%)$, correspondente à encontrada na literatura. ${ }^{1,6}$ Nos CA, a sobrevivência aos cinco anos foi de $66,7 \%$ também concordante com os dados obtidos em estudos anteriores. ${ }^{3,6}$ 
Não se verificaram diferenças na sobrevivência nos doentes submetidos a ressecção sublobar atípica em relação à ressecção lobar.

Achamos importante salientar que o facto de o estudo ter sido realizado no período compreendido entre 19992010 pode ser uma limitação do mesmo, dado o aparecimento recente de outros métodos diagnósticos e de estadiamento.

\section{CONCLUSÃO}

A nossa amostra exibiu características semelhantes às séries previamente reportadas. A maioria dos doentes apresentou-se em estadio ressecável e com excelente

\section{REFERÊNCIAS}

1. Escalon J, Detterbeck F, Shields T, LoCicero JI, Reed C, Feins R, editors. Carcinoid tumors. General thoracic surgery. $7^{\text {th }}$ ed. Philadelphia: Lippincott Williams \& Wilkins; 2009. p. 1539-54.

2. Bertino EM, Confer FD, Colonna JE, Ross P, Otterson GA. Pulmonary neuroendocrine/carcinoid tumors. Cancer. 2009;115:4434-41.

3. Travis WD, Brambilla E, Muller-Hermelink HK, Harris CC. Pathology and genetics of tumors of the lung, pleura, thymus and heart. Lyon: IARC Press; 2004.

4. Detterbeck FC. Management of carcinoid tumors. Ann Thorac Surg. 2010;89:998-1005

5. Bini A, Brandolini J, Cassanelli N, Davoli F, Dolci G, Sellitri F, et al. Typical and atypical pulmonary carcinoids: our institutional experience. Interact Cardiovasc Thorac Surg. 2008;7:415-8.

6. Ferguson MK, Landreneau RJ, Hazelrigg SR, Altorki NK, Naunheim KS, Zwischenberger JB, et al. Longterm outcome after resection for bronchial carcinoid tumors. Eur J Cardiothorac Surg. 2000;18:156-61.

7. Harpole DH Jr, Feldman JM, Buchanan S, Young G, Wolfe WG. Bronchial carcinoid tumors: a retrospective analysis of 126 patients. Ann Thorac Surg. 1992;54:50-5.

8. Garcia-Yuste M, Matilla JM, Cueto A, Paniagua JM, Ramos G, Canizares MA, et al. Typical and atypical carcinoid tumors: analysis of the experience of the Spanish Multi-centric Study of Neuroendocrine Tumours of the Lung. Eur J Cardiothorac Surg. 2007;31:192-7.

9. Rizzardi G, Marulli G, Bortolotti L, Calabrese F, Sartori F, Rea F. Sleeve resections and bronchoplastic procedures in typical central carcinoid tumours. Thorac Cardiovasc Surg. 2008;56:42-5.

10. Cao C, Yan TD, Kennedy C, Hendel N, Bannon PG, McCaughan BC Bronchopulmonary carcinoid tumors: long-term outcomes after resection. Ann Thorac Surg. 2011;91:339-3.

11. Skov BG, Krasnik M, Lantuejoul S, Skov T, Brambilla E. Reclassification of neuroendocrine tumors improves the separation of carcinoids and the prognóstico. O subtipo mais frequente foi o carcinóide típico sem envolvimento ganglionar. Os fatores relacionados com um pior prognóstico foram o subtipo histológico (CT vs. CA), o tamanho do tumor, o envolvimento ganglionar e a presença de metástases.

\section{CONFLITOS DE INTERESSE}

Os autores declaram não ter qualquer conflito de interesse relativamente ao presente artigo.

\section{FONTES DE FINANCIAMENTO}

Este trabalho não recebeu qualquer contribuição e subsídio ou bolsa.

prediction of survival. J Thorac Oncol. 2007;3:1410-5.

12. Sobin L, Gospodarowicz M, Wittekind C, editors. TNM classification of malignant Tumors. $7^{\text {th }}$ ed. Chichester: Wiley-Blackwell; 2009.

13. Travis WD, Giroux DJ, Chansky K, Crowley J, Asamura H, Brambilla E, et al. The IASLC Lung Cancer Staging Project: proposals for the inclusion of broncho-pulmonary carcinoid tumors in the fothcoming (seventh) edition of the TNM classification for Lung Cancer. J. Thorac Oncol. 2008;3:1213-33.

14. Filosso PL, Rena O, Donati G, Casadio C, Ruffini E, Papalia E, et al. Bronchial carcinoid tumors: surgical management and long-term outcome. J Thorac Cardiovasc Surg. 2002;123:303-9.

15. Travis WD, Rush W, Flieder DB, Falk R, Fleming MV, Gal AA, et al. Survival analysis of 200 pulmonary neuroendocrine tumors with clarification of criteria for atypical carcinoid and its separation from typical carcinoid. Am J Surg Pathol. 1998;22:934-44.

16. Naalsund A, Rostad H, Strøm EH, Lund MB, Strand TE. Carcinoid lung tumors- incidence, treatment and outcomes: a population-based study. Eur J Cardiothorac Surg. 2011;39:565-9.

17. Mezzetti M, Raveglia F, Panigalli T, Giuliani L, Lo Giudice F, Meda S, et al. Assessment of outcomes in typical and atypical carcinoids according to latest WHO classification. Ann Thorac Surg. 2003;76:1838-42.

18. Fink G, Krelbaum T, Yellin A, Bendayan D, Saute M, Glazer M, et al Pulmonary carcinoid: presentation, diagnosis, and outcome in 142 cases in Israel and review of 640 cases from the literature. Chest. 2001;119:1647-51.

19. Kurul IC, Topcu S, Tastepe I, Yazici U, Altinok T, Cetin G. Surgery in bronchial carcinoids: experience with 83 patients. Eur J Cardiothorac Surg. 2002;21:883-7.

20. Thomas CF Jr, Tazelaar HD, Jett JR. Typical and atypical carcinoids: outcome in patients presenting with regional lymph node involvement. Chest. 2001;119:1143-50. 


\section{Carcinóide Pulmonar: Análise de Experiência Institucional e Fatores Prognóstico}

Acta Med Port 2014:27:749-754

Publicado pela Acta Médica Portuguesa, a Revista Científica da Ordem dos Médicos

Av. Almirante Gago Coutinho, 151

1749-084 Lisboa, Portugal.

Tel: +351218428215

E-mail: submissao@actamedicaportuguesa.com

www.actamedicaportuguesa.com

ISSN:0870-399X | e-ISSN: 1646-0758

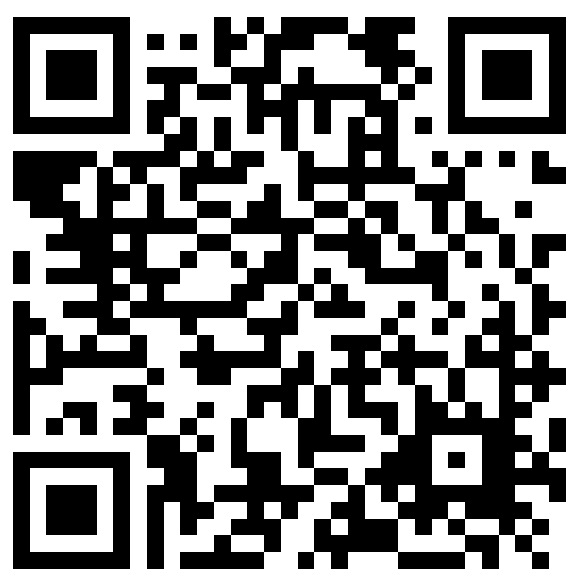

